\title{
Evolution of stellar winds from the Sun to red giants
}

\author{
Takeru K. Suzuki \\ School of Arts and Sciences, University of Tokyo, Komaba, Meguro, Tokyo, Japan 153-8902 \\ email: stakeru@ea.c.u-tokyo.ac.jp
}

\begin{abstract}
By performing global 1D MHD simulations, we investigate the heating and acceleration of solar and stellar winds in open magnetic field regions. Our simulation covers from photosphere to 20-60 stellar radii, and takes into account radiative cooling and thermal conduction. We do not adopt ad hoc heating function; heating is automatically calculated from the solutions of Riemann problem at the cell boundaries. In the solar wind case we impose transverse photospheric motions with velocity $\sim 1 \mathrm{~km} / \mathrm{s}$ and period between 20 seconds and 30 minutes, which generate outgoing Alfvén waves. We have found that the dissipation of Alfvén waves through compressive wave generation by decay instability is quite effective owing to the density stratification, which leads to the sufficient heating and acceleration of the coronal plasma. Next, we study the evolution of stellar winds from main sequence to red giant phases. When the stellar radius becomes $\sim 10$ times of the Sun, the steady hot corona with temperature $10^{6}$ $\mathrm{K}$, suddenly disappears. Instead, many hot and warm $\left(10^{5}-10^{6} \mathrm{~K}\right)$ bubbles are formed in cool $\left(T<2 \times 10^{4} \mathrm{~K}\right)$ chromospheric winds because of the thermal instability of the radiative cooling function; the red giant wind is not a steady stream but structured outflow.
\end{abstract}

Keywords. waves, MHD, Sun: photosphere, Sun: chromosphere, Sun: Corona, solar wind, stars: chromospheres, stars: coronae, stars: magnetic fields, stars: mass loss, stars: late-type

\section{Introduction}

Research on heliophysical processes covers broad fields from basic plasma physics to various applications such as space weather forecasts, which are widely discussed in this conference. One direction of heliophysics research is to understand the evolution of the solar system, or more generally, star(s)-planets systems.

For example, UV/X-ray radiations and winds from young stars affect the evolution of protoplanetary disks and the formation of planets. Recent observations have shown that younger stars are more active than main sequence stars, giving considerably larger X-ray and UV flux luminosity (Güdel 2004) and higher wind mass flux (Wood et al. 2005). We can also infer the properties of the early sun from observations of young solar-like stars (Güdel 2008). Current planet formation models are constructed by incorporating these observational facts.

Asterospheres of stars on red giant branch or later stages are also expected to play a significant role in the evolution of planets. The radius of a star expands after the end of main sequence phase. Close planets would be eventually engulfed. Before the engulfment, properties of atmosphere and wind change through the evolution; as inferred from observations of red giants, the wind mass flux increases considerably (Judge \& Stencel 1991), which could affect the evolution of planets. In this talk, we mainly introduce our own work on the evolution of stellar winds from the Sun to red giant.

In this proceeding, we firstly summarize our self-consistent MHD simulations of solar winds from the photosphere to sufficiently outer region (Suzuki \& Inutsuka 2005; 2006; hereafter AI05 \& SI06). We introduce a scaling of the solar wind speeds near $1 \mathrm{AU}$ based on our simulations (Suzuki 2006). Then, we discuss the evolution of stellar winds from the Sun to red giant stars (Suzuki 2007). 


\section{Solar wind}

\subsection{Simulation set-up}

We consider 1D open flux tubes which are super-radially open, measured by heliocentric distance, $r$. The simulation regions are from the photosphere $\left(r=1 R_{\odot}\right)$ with density, $\rho=10^{-7} \mathrm{~g}$ $\mathrm{cm}^{-3}$ to $65 R_{\odot}(0.3 \mathrm{AU})$. Radial field strength, $B_{r}$, is given by conservation of magnetic flux as $B_{r} r^{2} f(r)=$ const., where $f(r)$ is a super-radial expansion factor. For the fast solar wind case, we adopt $B_{r, 0}=161 \mathrm{G}$ at the photosphere and the total expansion factor $=75$ (see SI05 and SI06 for detail).

We input the transverse fluctuations of the field line by the granulations at the photosphere, which excite Alfvén waves. In this paper we only show results of linearly polarized perturbations with power spectrum proportional to $1 / \nu$, where $\nu$ is frequency (for circularly polarized fluctuations with different spectra, see SI06). Amplitude, $\left\langle d v_{\perp, 0}\right\rangle$, at the photosphere is chosen to be compatible with the observed photospheric velocity amplitude $=0.7 \mathrm{~km} \mathrm{~s}^{-1}$ (Holweger et al. 1978). At the outer boundaries, outgoing condition is imposed for all the MHD waves, which enables us to carry out simulations for a long time until quasi-steady state solutions are obtained without unphysical wave reflection.

We dynamically treat the propagation and dissipation of the waves and the heating and acceleration of the plasma by solving ideal MHD equations. In the energy equation we take into account radiative cooling and Spitzer thermal conduction (SI06). We adopt the second-order MHD-Godunov-MOCCT scheme (Sano \& Inutsuka 2008 in preparation) to update the physical quantities. We initially set static atmosphere with a temperature $T=10^{4} \mathrm{~K}$ to see whether the atmosphere is heated up to coronal temperature and accelerated to accomplish the transonic flow. At $t=0$ we start the inject of the transverse fluctuations from the photosphere and continue the simulations until the quasi-steady states are achieved.

\subsection{Results of simulation}

Figure 1 plots the initial condition (dashed lines) and the results after the quasi-steady state condition is achieved at $t=2573$ minutes (solid lines), compared with recent observations of fast solar winds. Figure 1 shows that the initially cool and static atmosphere is effectively heated and accelerated by the dissipation of the Alfvén waves. The sharp transition region which divides the cool chromosphere with $T \sim 10^{4} \mathrm{~K}$ and the hot corona with $T \sim 10^{6} \mathrm{~K}$ is formed owing to a thermally unstable region around $T \sim 10^{5} \mathrm{~K}$ in the radiative cooling function (Landini \& Monsignori-Fossi 1990). The hot corona expands outward as the transonic solar wind. The simulation naturally explains the observed trend quite well.

The heating and acceleration of the solar wind plasma in inner heliosphere is done by the dissipation of Alfvén waves. Here we inspect waves in more detail. Figure 2 presents contours of amplitude of $v_{r}, \rho, v_{\perp}$, and $B_{\perp} / B_{r}$ in $R_{\odot} \leqslant r \leqslant 15 R_{\odot}$ from $t=2570 \mathrm{~min}$. to $2600 \mathrm{~min}$. Blue (gray) shaded regions denote positive (negative) amplitude. Above the panels, we indicate the directions of the local 5 characteristics, two Alfvén, two slow, and one entropy waves at the respective positions. Note that the fast MHD and Alfvén modes degenerate in our case (wave vector and underlying magnetic field are in the same direction), so we simply call the innermost and outermost waves Alfvén modes. In our simple 1D geometry, $v_{r}$ and $\rho$ trace the slow modes which have longitudinal character, while $v_{\perp}$ and $B_{\perp}$ trace the Alfvén modes which are transverse.

Alfvén waves areseen in in $v_{\perp}$ and $B_{\perp} / B_{r}$ diagrams, which have the same slopes with the Alfvén characteristics shown above. One can also find the incoming waves propagating from lower-right to upper-left as well as the outgoing modes generated from the surface. These incoming waves are generated by the reflection at the 'density mirrors' of the slow modes. At intersection points of the outgoing and incoming characteristics the non-linear wave-wave interactions take place, which play a role in the wave dissipation.

The slow modes appears in $v_{r}$ and $\rho$ diagrams. After inspecting the diagrams we find that the most of the patterns are due to the outgoing slow modes $\dagger$ which are generated from the

$\dagger$ The phase correlation of the longitudinal slow waves is opposite to that of the transverse 

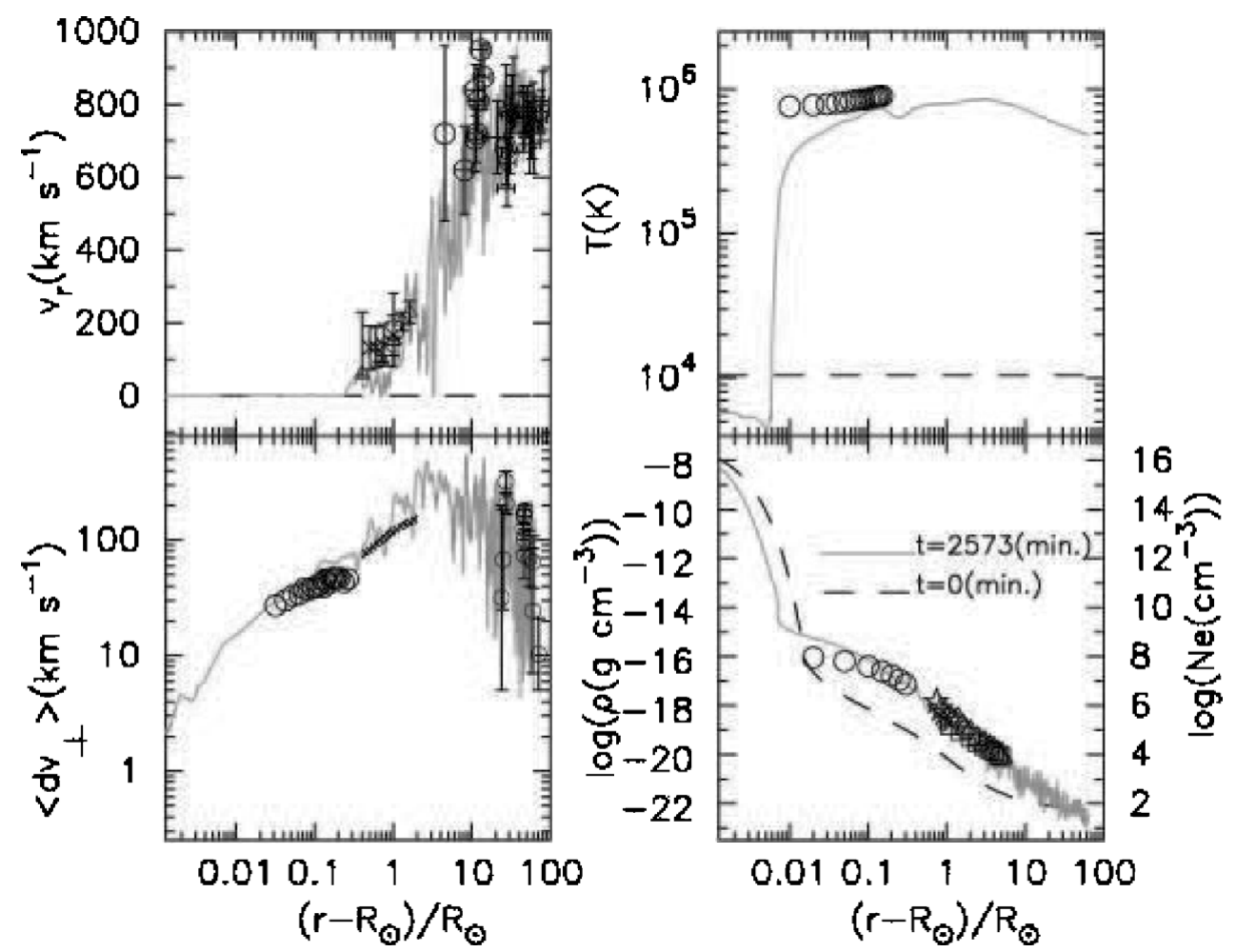

Figure 1. Results of fast solar wind mode with observations in polar regions. Outflow speed, $v_{r}\left(\mathrm{~km} \mathrm{~s}^{-1}\right)$ (top-left), temperature, $T(\mathrm{~K})$ (top-right), density in logarithmic scale, $\log \left(\rho\left(\mathrm{g} \mathrm{cm}^{-3}\right)\right)$ (bottom-right), and rms transverse amplitude, $\left\langle d v_{\perp}\right\rangle\left(\mathrm{km} \mathrm{s}^{-1}\right)$ (bottom-left) are plotted. Observational data in the third panel are electron density, $\log \left(N_{e}\left(\mathrm{~cm}^{-3}\right)\right)$ which is to be referred to the right axis. Dashed lines indicate the initial conditions and solid lines are the results at $t=2573$ minutes. In the bottom panel, the initial value $\left(\left\langle d v_{\perp}\right\rangle=0\right)$ dose not appear. The observational data in the inner region $\left(<6 R_{\odot}\right)$ are from SOHO (Teriaca et al. 2003; Zangrilli et al. 2002; Fludra et al. 1999; Wilhelm et al. 1998; Lamy et al. 1997; Banergee et al. 1998; Esser et al. 1999) and those in the outer region are from interplanetary scintillation measurements (Grall et al. 1996; Habbal et al. 1994; Kojima et al. 2004; Canals et al. 2002).

perturbations of the Alfvén wave pressure, $B_{\perp}^{2} / 8 \pi$ (Kudoh \& Shibata 1998\& Tsurutani et al. 2002). These slow waves steepen eventually result in the shock dissipation.

Figure 3 presents the dissipation of the waves more quantitatively. We plot so-called wave actions,

$$
S_{c}=\rho \delta v^{2} \frac{\left(v_{r}+v_{\mathrm{ph}}\right)^{2}}{v_{\mathrm{ph}}} \frac{r^{2} f(r)}{r_{c}^{2} f\left(r_{c}\right)},
$$

of outgoing Alfvén, incoming Alfvén, and outgoing slow MHD (sound) waves, where $\delta v$ and $v_{\mathrm{ph}}$ are amplitude and phase speed of each wave mode. $S_{c}$ is an adiabatic constant derived from wave action (Jacques 1977) in unit of energy flux. For the incoming Alfvén wave, we plot the opposite sign of $S_{c}$ so that it becomes positive in the sub-Alfvénic region. The outgoing and incoming Alfvén waves are decomposed by correlation between $v_{\perp}$ and $B_{\perp}$. Extraction of the slow wave is also from fluctuating components of $v_{r}$ and $\rho$.

Figure 3 exhibits that the outgoing Alfvén waves dissipate quite effectively; $S_{c}$ becomes only $\sim 10^{-3}$ of the initial value at the outer boundary. A sizable amount is reflected back downward

Alfvén waves. The outgoing slow modes have the positive correlation between amplitudes of $v_{r}$ and $\rho,\left(\delta v_{r} \delta \rho>0\right)$, while the incoming modes have the negative correlation $\left(\delta v_{r} \delta \rho<0\right)$. 


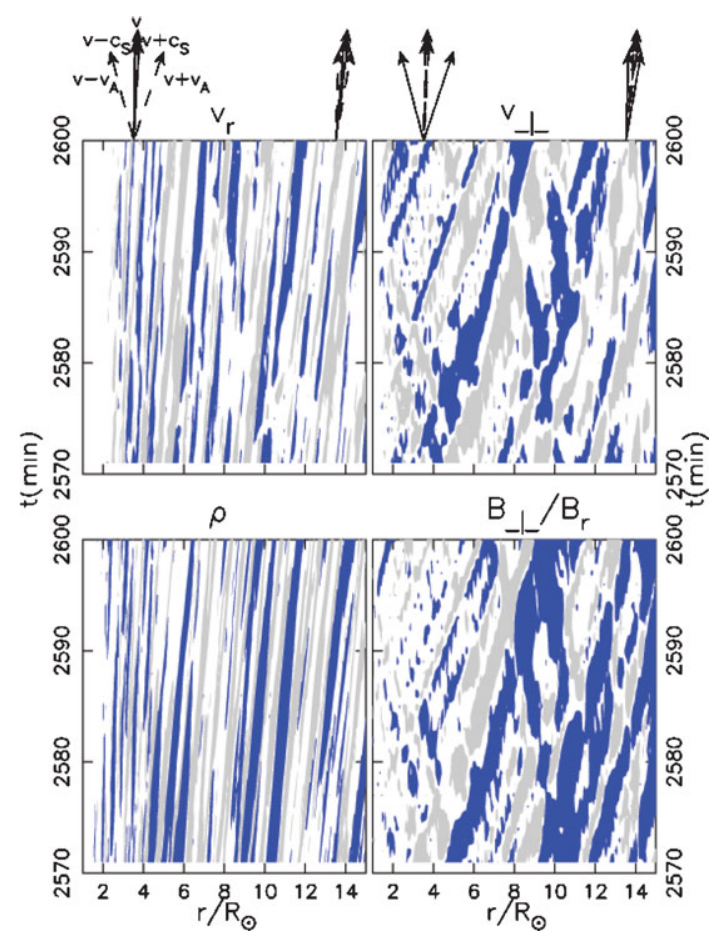

Figure 2. $r-t$ diagrams for $v_{r}$ (upper-left), $\rho$ (lower-left), $v_{\perp}$ (upper-right), and $B_{\perp} / B_{r}$ (lowerright.) The horizontal axises cover from $R_{\odot}$ to $15 R_{\odot}$, and the vertical axises cover from $t=2570$ minutes to 2600 minutes. Dark(blue) and light shaded regions indicate positive and negative amplitudes which exceed certain thresholds. The thresholds are $d v_{r}= \pm 96 \mathrm{~km} / \mathrm{s}$ for $v_{r}, d \rho / \rho= \pm 0.25$ for $\rho, v_{\perp}= \pm 180 \mathrm{~km} / \mathrm{s}$ for $v_{\perp}$, and $B_{\perp} / B_{r}= \pm 0.16$ for $B_{\perp} / B_{r}$, where $d \rho$ and $d v_{r}$ are differences from the averaged $\rho$ and $v_{r}$. Arrows on the top panels indicate characteristics of Alfvén, slow MHD and entropy waves at the respective locations.

below the coronal base $\left(r-R_{\mathrm{S}}<0.01 R_{\mathrm{S}}\right)$, which is known from the incoming Alfvén wave following the outgoing component with slightly smaller level. This is because the wave shape is considerably deformed owing to the steep density gradient; a typical variation scale $\left(<10^{5} \mathrm{~km}\right)$ of the Alfvén speed becomes comparable to or even shorter than the wavelength $\left(=10^{4}-10^{6} \mathrm{~km}\right)$. Although the energy flux, $\simeq 5 \times 10^{5} \mathrm{erg} \mathrm{cm}^{-2} \mathrm{~s}^{-1}$, of the outgoing Alfvén waves $\left(S_{c}\right.$ in the static region is equivalent with the energy flux) which penetrates into the corona is only $\simeq 15 \%$ of the input value, it is sufficient for the energy budget in the coronal holes (Withbroe \& Noyes 1977).

The processes discussed here are the combination of the direct mode conversion to the compressive waves and the parametric decay instability due to three-wave (outgoing Alfvén, incoming Alfvén, and outgoing slow waves) interactions (Goldstein 1978; Terasawa et al. 1986) of the Alfvén waves. These processes, which are not generally efficient in homogeneous background, become effective by amplification of velocity amplitude in the density decreasing atmosphere. The Alfvén speed also changes even within one wavelength of Alfvén waves with periods of minutes. This leads to both variation of the wave pressure in one wavelength and partial reflection through the deformation of the wave shape (Moore et al. 1991). The density stratification plays a key role in the propagation and dissipation of the Alfvén waves.

\subsection{Solar wind speed}

It is widely believed that properties of open flux tubes are important parameters that control the solar wind speed. Wang \& Sheeley $(1990 ; 1991)$ showed that the solar wind speed at $\sim 1 \mathrm{AU}$ is anti-correlated with a super-radial expansion factor, $f_{\text {tot }}$, from their long-term observations as well as by a simple theoretical model. Ofman \& Davila (1998) showed this tendency by 


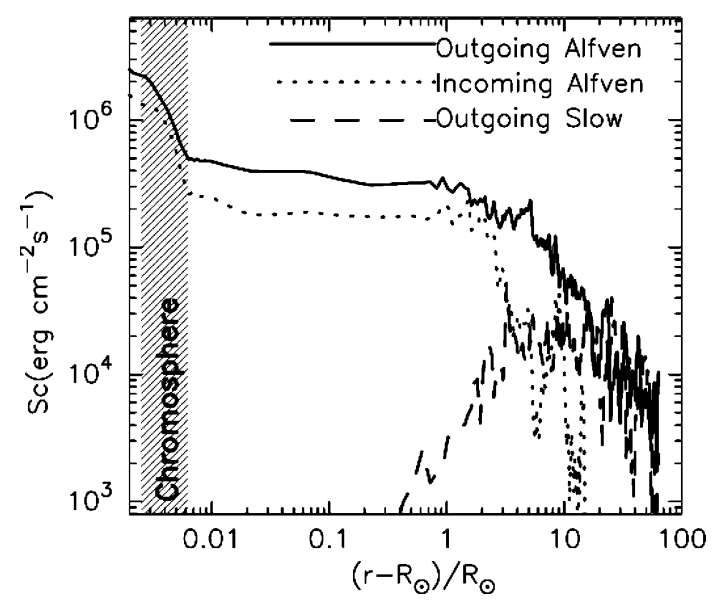

Figure 3. $S_{c}$ of outgoing Alfvén mode (solid), incoming Alfvén mode (dotted), and outgoing MHD slow mode (dashed) at $t=2573$ mins. Hatched region indicates the chromosphere and low transition region with $T<4 \times 10^{4} \mathrm{~K}$.

time-dependent simulations as well. Fisk et al. (1999) claimed that the wind speed should be positively correlated with photospheric field strength, $B_{r, 0}$, by a simple energetics consideration.

Kojima et al. (2005) have found that the solar wind velocity is better correlated with the combination of these two parameters, $B_{r, 0} / f_{\mathrm{tot}}$, than $1 / f_{\mathrm{tot}}$ or $B_{r, 0}$ from the comparison of the outflow speeds obtained by their interplanetary scintillation measurements with the corresponding flux tubes (Figure 4). Suzuki $(2004 ; 2006)$ and SI06 also pointed out that $B_{r, 0} / f_{\text {tot }}$ should be the best control parameter provided that the Alfvén waves play a dominate role in the coronal heating and the solar wind acceleration. This is because the nonlinearity of the Alfvén waves, $\left\langle\delta v_{\mathrm{A},+}\right\rangle / v_{\mathrm{A}}$ is controlled by $v_{\mathrm{A}} \propto B_{r} \propto B_{r, 0} / f_{\text {tot }}$ in the outer region where the flux tube is already super-radially open. Wave energy does not effectively dissipate in the larger $B_{r, 0} / f_{\text {tot }}$ case in the subsonic region because of relatively small nonlinearity and more energy remains in the supersonic region. In general, energy and momentum inputs in the supersonic region gives higher wind speed, while those in the subsonic region raises the mass flux $\left(\rho v_{r}\right)$ of the wind by an increase of the density (Lamers \& Cassinelli 1999). This indicates that the solar wind speed is positively correlated with $B_{r, 0} / f_{\text {tot }}$.

Suzuki (2006) further derived a relation between the solar wind speed, $v_{1 \mathrm{AU}}$, at $1 \mathrm{AU}$ and surface properties from a simple energetics argument:

$$
\begin{aligned}
v_{1 \mathrm{AU}} & =\left[2 \times\left(-\frac{R_{\odot}^{2}}{4 \pi\left(\rho v r^{2}\right)_{1 \mathrm{AU}}} \frac{B_{r, \odot}}{f_{\mathrm{tot}}}\left\langle\delta B_{\perp} \delta v_{\perp}\right\rangle_{\odot}+\frac{\gamma}{\gamma-1} R T_{\mathrm{C}}-\frac{G M_{\odot}}{R_{\odot}}\right)\right]^{1 / 2} \\
& =300(\mathrm{~km} / \mathrm{s})\left[5.9\left(\frac{-\left\langle\delta B_{\perp} \delta v_{\perp}\right\rangle_{\odot}}{8.3 \times 10^{5}\left(\mathrm{~cm} \mathrm{~s}^{-1} \mathrm{G}\right)}\right)\left(\frac{B_{r, \odot}(\mathrm{G})}{f_{\mathrm{tot}}}\right)+3.4\left(\frac{\gamma}{1.1}\right)\left(\frac{0.1}{\gamma-1}\right)\left(\frac{T_{\mathrm{C}}}{10^{6}(\mathrm{~K})}\right)-4.2\right]^{1 / 2}
\end{aligned}
$$

where we have the three free parameters, surface amplitude, $\left\langle\delta B_{\perp} \delta v_{\perp}\right\rangle_{\odot}$, effective coronal temperature, $T_{\mathrm{C}}$, and ratio of specific heats, $\gamma$. In the equation, these parameters are evaluated by the standard values, which should be used for actual prediction of wind speed. The first term is the contribution from Alfvén waves, the second term represents net heating minus cooling due to radiation loss and thermal conduction, and the third term is the gravitational loss. The first term, due to Alfvén waves, exhibits the dependence on $B_{r, 0} / f_{\text {tot }}$, which reflects the Alfvén waves in expanding flux tubes. In previous works this term is neglected (Fisk et al. 1999) or more simplified (Schwadron \& McComas 2003) because this is a nonlinear term. However, on account of the dissipationless character of Alfvén waves it plays a role in the solar wind in spite of the nonlinear term. Figure 4 shows the prediction of Equation (2.2), in comparison with the observation using interplanetary scintillation measurements by Kojima et al. (2005). The observed data are nicely explained by the relation based on the simple energetics. 


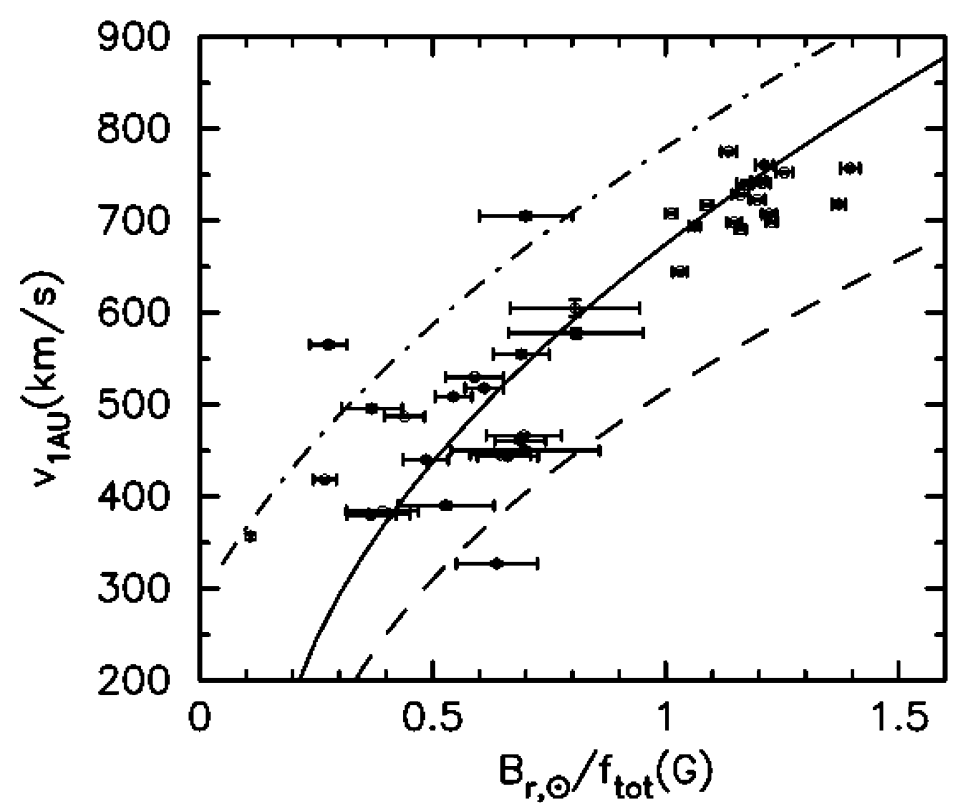

Figure 4. Relations between $v_{1 \mathrm{AU}}$ and $B_{r, \odot} / f_{\text {tot }}$. Lines are theoretical prediction from Equation (2.2). Solid line indicates the fiducial case $\left(\left\langle\delta B_{\perp} \delta v_{\perp}\right\rangle=8.3 \times 10^{5} \mathrm{G} \mathrm{cm} \mathrm{s}{ }^{-1}\right.$ and $\left.T_{\mathrm{C}}=10^{6} \mathrm{~K}\right)$. Dot-dashed line adopt higher coronal temperature $\left(T_{\mathrm{C}}=1.5 \times 10^{6} \mathrm{~K}\right)$ with the fiducial $\left\langle\delta B_{\perp} \delta v_{\perp}\right\rangle$. Dashed line adopt smaller $\left\langle\delta B_{\perp} \delta v_{\perp}\right\rangle\left(=5.3 \times 10^{5} \mathrm{G} \mathrm{cm} \mathrm{s}^{-1}\right)$ with the fiducial temperature. Observed data are from Kojima et al. (2005). Coronal magnetic fields are extrapolated from $B_{r, \odot}$ by the potential field-source surface method Hakamada \& Kojima (1999). $f_{\text {tot }}$ is derived from comparison between the areas of open coronal holes at the photosphere and at the source surface $\left(r=2.5 R_{\odot}\right) . v_{1 \mathrm{AU}}$ is obtained by interplanetary scintillation measurements. $v_{1 \mathrm{AU}}, B_{r, \odot}$, and $f_{\text {tot }}$ are averaged over the area of each coronal hole and the data points correspond to individual coronal holes.

\section{Evolution to red giants}

We investigate the evolution of stellar winds with stellar evolution to red giants. Red giant stars, similarly to the Sun, generally possess surface convective layers. Also, red giants are slow rotators as a result of angular momentum loss (magnetic braking) through stellar evolution. Then, our solar wind simulation is directly applicable to red giant winds. We would also like to note that our simulation can be applied to winds from young stars, such as proto-stars and $\mathrm{T}$ Tauri stars, because they are expected to be driven mainly by surface turbulence as well, whereas the effects of accretion (Cranmer 2008) and rotation might have to be taken into account in these objects.

We consider winds from $1 M_{\odot}$ and $3 M_{\odot}$ stars in various evolutionary stages from the main sequence to the red giant branch. The properties of surface fluctuations (e.g. amplitude and spectrum) can be estimated from conditions of surface convection which is negatively correlated with surface gravity and positively correlated with temperature (e.g. Renzini et al. 1977; Stein et al. 2004). Because there are very few observations of the magnetic fields, we simply use the same surface strength $B_{r, 0}=240 \mathrm{G}$ and super-radial expansion factor, $f_{\text {tot }}=240$, for all the cases. Then, we carry out the simulations of the red giant winds in a similar manner to the solar wind simulations.

Figure 5 presents the evolution of stellar winds of a $1 M_{\odot}$ star from main sequence to red giant stages. The middle panel shows that the average temperature drops suddenly from $T \simeq 7 \times 10^{5} \mathrm{~K}$ in the sub-giant star (dash-dotted) to $T \leqslant 10^{5} \mathrm{~K}$ in the red giant stars, which is consistent with the observed "dividing line" (Linsky \& Haisch 1979). The main reason of the disappearance of the steady hot coronae is that the sound speed $\left(\approx 150 \mathrm{~km} \mathrm{~s}^{-1}\right)$ of $\approx 10^{6} \mathrm{~K}$ plasma exceeds the escape speed, $v_{\text {esc }}(r)=\sqrt{2 G M_{\star} / r}$, at $r \gtrsim$ a few $R_{\star}$ in the red giant stars; the hot corona cannot be confined by the gravity any more in the atmospheres of the red giant stars. Therefore, the 


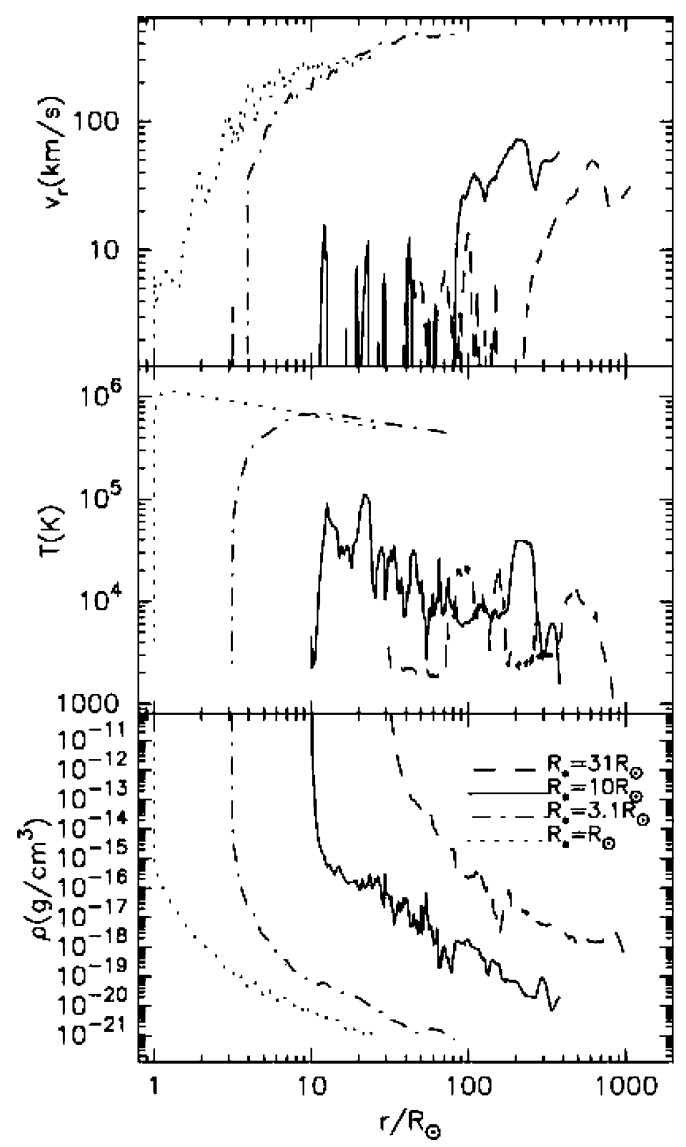

Figure 5. Time-averaged stellar wind structure of the $1 M_{\odot}$ stars. From the top to the bottom, radial outflow velocity, $v_{r}\left(\mathrm{~km} \mathrm{~s}^{-1}\right)$, temperature, $T(\mathrm{~K})$, and density, $\rho\left(\mathrm{g} \mathrm{cm}^{-3}\right)$, are plotted. The dotted, dash-dotted, solid, and dashed lines are the results of stellar radii, $R=R_{\odot}$ (the present Sun), 3.1 $R_{\odot}$ (sub-giant), $10 R_{\odot}$ (red giant), and $31 R_{\odot}$ (red giant), respectively.

material flows out before heated up to coronal temperature. In addition, the thermal instability of the radiative cooling function (Landini \& Monsignori-Fossi 1990) plays a role in the sudden decrease of temperature; since the gas with $T=10^{5}-\lesssim 10^{6} \mathrm{~K}$ is unstable, the temperature quickly decreases from the subgiant to red giants.

The densities of the winds increase with stellar evolution due to the decrease of the surface gravity. On the other hand, the velocities of the winds decrease because slower winds can escape from the stellar gravity in low-gravity evolved stars. The mass loss rate increases due to the increase of the stellar surface $\left(\propto R^{2}\right)$ and the increase of the density. From the sun to the star with $R_{\star}=31 R_{\odot}$, the mass loss rate increases $10^{5}-10^{6}$ times. The physical properties at $1 \mathrm{AU}$ greatly change for the red giant Sun: for the $R_{\star}=31 R_{\odot}$ star the density is $10^{8}\left(1 / \mathrm{cm}^{3}\right)$, which is $\sim 10^{7}$ times larger than the current value and the wind velocity is very slow $\sim 10 \mathrm{~km} / \mathrm{s}$ and still in the acceleration region. The wind temperature mostly $\lesssim 10^{4} \mathrm{~K}$ in chromospheric winds, but hot bubbles with $\gtrsim 10^{5}-10^{6} \mathrm{~K}$ sometimes pass through, which we discuss below.

The thermal instability also results in structured and time-dependent stellar winds from red giant stars. Figure 6 shows the snap-shot wind structure of a $3 M_{\odot}$ red giant star at $t=6909(\mathrm{hr})$ (solid) in comparison with the time-averaged structure (dashed). Figure 6 illustrates that the simple picture of layered atmosphere, photosphere - chromosphere - transition region - corona wind from the stellar surface, does not hold in red giant stars. A characteristic feature is that a number of hot bubbles with low densities are distributed in cool background material. For example, in an inner region $\left(r-R_{\star}<R_{\star}\right)$ the two bubbles with the peak temperatures $>10^{6}$ $\mathrm{K}$ are formed; in $R_{\star}<r-R_{\star} \lesssim 10 R_{\star}$ a couple of warm bubbles with $T \gtrsim 10^{5} \mathrm{~K}$ are formed. 


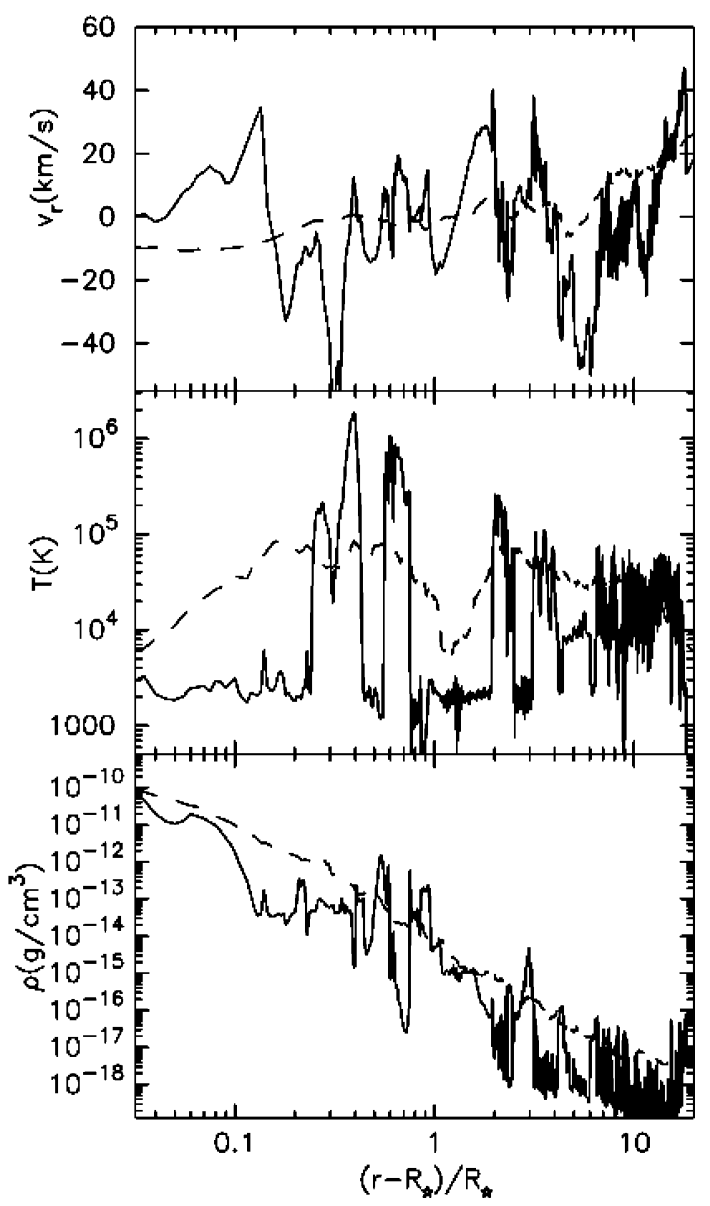

Figure 6. Snap-shot wind structure (solid) of a $3 M_{\odot}$ red giant star with surface gravity $\log g=1.4$ at $t=6909(\mathrm{hr})$, compared with the time-averaged structure (dashed). From the top to the bottom, $v_{r}\left(\mathrm{~km} \mathrm{~s}^{-1}\right), T(\mathrm{~K})$, and $\rho\left(\mathrm{g} \mathrm{cm}^{-3}\right)$ are plotted on $\left(r-R_{\star}\right) / R_{\star}$.

The hot and warm bubbles and the cool background materials are connected by the transition regions, at which the temperature drastically changes because of the thermal instability. These hot bubbles are also seen in $1 M_{\odot}$ red giant stars.

The densities of the hot bubbles are lower than the ambient media to satisfy the pressure balance. As seen in the bottom panel of Figure 6 the density fluctuates typically by 2-3 orders of magnitude in the wind, which is related to the fact that the gas mainly consists of hot $\left(>10^{6} \mathrm{~K}\right)$ and cool $\left(<10^{4} \mathrm{~K}\right)$ components $\dagger$. The outflow speed fluctuates as well to fulfill the mass conservation relation. The red giant wind is not a steady outward stream but an outflow consisting of many small-scale structures. Our simulations show that both hot plasma and cool chromospheric wind coexist in red giant stars, which is consistent with reported hybrid activities (Hartmann et al. 1980, Harper et al. 1995, Ayres et al. 1998).

\section{Summary \& discussions}

We have studied the evolution of stellar wind from the Sun to red giants by performing 1D MHD numerical simulations. The Alfvén waves are generated by the footpoint fluctuations of the magnetic field lines. We have treated the wave propagation and dissipation, and the heating

$\dagger$ Strictly speaking, however, magnetic pressure also needs to be taken into account for the force balance (Suzuki 2007). 
and acceleration of the plasma in a self-consistent manner. Our simulation is the first simulation which treats the wind from the photosphere to the (inner) heliosphere with the relevant physical processes.

We have shown that the dissipation of the Alfvén waves through the generation of the compressive waves (decay instability) and shocks (nonlinear steepening) is one of the solutions for the heating and acceleration of the plasma in the coronal holes. However, we should cautiously examine the validity of the 1-D MHD approximation we adopt. There are other dissipation mechanisms due to the multidimensionality (e.g. Ofman 2004), such as turbulent cascade into the transverse direction (Goldreich \& Sridhar 1995; Oughton et al. 2001) and phase mixing (Heyvaerts \& Priest 1983). If Alfvén waves cascade to higher frequency, kinetic effects (e.g. Axford \& McKenzie 1997; Nariyuki \& Hada 2006) becomes important.

We have also extended the solar wind simulations to red giant winds. With stellar evolution, the steady hot corona with temperature, $T \approx 10^{6} \mathrm{~K}$, suddenly disappears because the surface gravity becomes small; hot plasma cannot be confined by the gravity. Thermal instability also generate intermittent magnetized hot bubbles in cool chromospheric winds. When the solar radius expands $\sim 30$ times, the earth orbit is still in the wind acceleration region and the wind density is $10^{7}$ times larger than the present value there. In the red giant simulations, we assume the same photospheric field strength and super-radial expansion factor because very little information is known from observations at the moment. It is important to study the evolution of magnetic fields with stellar evolution from both observation and modelling.

\section{Acknowledgements}

The author thanks the organizers of IAU257 for the nice conference. This work is supported in part by Inamori Foundation and a Grant-in-Aid for Scientific Research (19015004 \& 20740100) from the Ministry of Education, Culture, Sports, Science, and Technology of Japan.

\section{References}

Axford, W. I. \& McKenzie, J. F. 1997 The Solar Wind in "Cosmic Winds and the Heliosphere", Eds. Jokipii, J. R., Sonnet, C. P., and Giampapa, M. S., University of Arizona Press, 31

Ayres, T. R., Simon, T., Stern, R. A., Drake, S. A., Wood, B. E., \& Brown, A. 1998 ApJ, 496, 428

Banerjee, D., Teriaca, L., Doyle, J. G., \& Wilhelm, K. 1998 A\&A, 339, 208

Canals, A., Breen, A. R., Ofman, L., Moran, P. J., \& Fallows, R. A., 2002 Ann. Geophys., 20, 1265

Cranmer, S. R. 2008, ApJ in press (arxiv0808.2250)

Esser, R., Fineschi, S., Dobrzycka, D., Habbal, S. R., Edgar, R. J., Raymond, J. C., \& Kohl, J. L., 1999 ApJ, 510, L63

Fisk, L. A., Schwadron, N. A., \& Zurbuchen, T. H. J. Geophys. Res., 104, A4, 19765

Fludra, A., Del Zanna, G., \& Bromage, B. J. I., 1999 Spa. Sci. Rev., 87, 185

Goldreich, P. \& Sridhar, S., 1995 ApJ, 438, 763

Goldstein, M. L., 1978, ApJ, 219, 700

Grall, R. R., Coles, W. A., Klinglesmith, M. T., Breen, A. R., Williams, P. J. S., Markkanen, J., \& Esser, R., 1996 Nature, 379, 429

Güdel, M. 2004, ARA\&A, 12, 71

Güdel, M. 2007, Living Rev. in Sol. Phys., 4, 3

Habbal, S. R., Esser, R., Guhathakura, M., \& Fisher, R. R., 1994 Gephys. Res. Lett., 22, 1465 Hakamada, K. \& Kojima, M. 1999, Sol. Phys., 187, 115

Harper, G. M., Wood, B. E., Linsky, J. L., Bennett, P. D., Ayres, T. R., \& Brown, A. 1995, ApJ, 452, 407

Hartmann, L., Dupree, A. K., \& Raymond, J. C. 1980, ApJ, 236, L143

Heyvaerts, J. \& Priest, E. R., 1983 A\&A, 117, 220

Holweger, H., Gehlsen, M., \& Ruland, F., 1978 A\&A, 70, 537

Jacques, S. A. 1977, ApJ, 215, 942

Judge, P. G. \& Stencel, R. E. 1991, ApJ, 371, 357

Kojima, M., Breen, A. R., Fujiki, K., Hayashi, K., Ohmi, T., \& Tokumaru, M., 2004 J. Geophys. Res., 109, A04103 
Kudoh, T. \& Shibata, K., 1999 ApJ, 514, 493

Lamers, H. J. G. L. M. \& Cassinelli, J. P. (1999), 'Introduction to Stellar Wind', Cambridge

Lamy, P., Quemerais, E., Liebaria, A., Bout, M., Howard, R., Schwenn, R., \& Simnett, G., 1997 Fifth SOHO Worshop, The Corona and Solar Wind near Minimum Activity, ed A. Wilson (ESA-SP 404; Noordwijk:ESA), 491

Landini, M. \& Monsignori-Fossi, B. C., 1990 A\&AS, 82, 229

Linsky, J. L. \& Haisch, B. M., 1979 ApJ, 229. L27

Moore, R. L., Suess, S. T., Musielak, Z. E., \& An, A.-H., 1991 ApJ, 378, 347

Nariyuki, Y. \& Hada, T., 2006 Phys. Plasma, 13, 124501

Ofman, L. 2004, J. Geophys. Res., 109, A07102

Ofman, L. \& Davila, J. M. 1998, J. Geophys. Res., 103, 23677

Oughton, S., Matthaeus, W. H., Dmitruk, P., Milano, L. J., Zank, G. P., \& Mullan, D. J., 2001 ApJ 551, 565

Renzini, A., Cacciari, C., Ulmschneider, P., \& Schmitz, F., 1977 A\&A, 61, 39

Schwadron, N. A. \& McComas, D. J. 2003 ApJ, 599, 1395

Stein, R. F., Georgobiani, D., Trampedach, R., Ludwig, H.-G., \& Nortlund, Å., 2004, Sol. Phys., 220,229

Suzuki, T. K., 2004 MNRAS, 349, 1227

Suzuki, T. K. 2006, ApJ 640, L75

Suzuki, T. K., 2007 ApJ, 659, 1592

Suzuki, T. K. \& Inutsuka, S., 2005 ApJ, 632, L49

Suzuki, T. K. \& Inutsuka, S., 2006 J. Geophys. Res., 111, A6, A06101

Terasawa, T., Hoshino, M., Sakai, J. I., \& Hada, T., 1986, J. Geophys. Res., 91, 4171

Teriaca, L., Poletto, G., Romoli, M., \& Biesecker, D. A., 2003 ApJ, 588, 566

Tsurutani, B. T. et al., 2002 Geophys. Res. Lett., 29, 23-1

Wang, Y.-M. \& Sheeley, Jr, N. R.: Solar wind speed and coronal flux-tube expansion, Astrophys. J., 355, 726-732, 1990

Wang, Y.-M. \& Sheeley, Jr, N. R.: Why fast solar wind originates from slowly expanding coronal flux tubes, Astrophys. J. Lett., 372, L45 - L48, 1991

Wilhelm, K., Marsch, E., Dwivedi, B. N., Hassler, D. M., Lemaire, P., Gabriel, A. H., \& Huber, M. C. E., 1998 ApJ, 500, 1023

Withbroe, G. L. \& Noyes, R. W., 1977, ARAA, 15, 363

Wood, B. E., Müller, H.-R., Zank, G. P., Linsky, J. L., \& Redfield, S. 2005, ApJ, 628, L143

Zangrilli, L., Poletto, G., Nicolosi, P., Noci, G., \& Romoli, M., 2002 ApJ, 574, 477

\section{Discussion}

ShiBATA: Very nice work! I have two questions: 1) Sometimes very low density solar wind is observed. Can you explain such low density solar wind? 2) As for red giant wind, how did you assume magnetic field distribution and strength?

Suzuki: 1) Non linear effects should be employed (Suzuki and Inutsuku 2006), but because it is complicated, I will discuss this issue with you later 2) I'm using the same B strength (240 G) in the photosphere and same super-radial expansion factor $(\sim 240 \mathrm{G})$. This is just an assumption.

ARGE: Please elaborate on your comment regarding that Alfven wave term not properly considered in previous work.

SuzukI: The new point of my work is that I include the Alfven wave term. This is a nonlinear term, but important in solar wind region because Alfven waves propagate through a long distance.

BOCHSLER: How does stellar rotation influence winds of red giants?

SuzuKI: The stellar rotation of red giants is negligible in terms of wind acceleration because red giants are slow rotators. But if we consider younger stars, the rotation may be important. 
JARDINE: Just a comment. While I agree that centrifugal effects may not be important in slow rotators, the effect of rotation rate on the strength of the magnetic field that is generated is a more serious concern.

SuZUKI: I agree with the comment. Thank you.

Ofman: 1. Have you considered the effect of ion-neutral dissipation on Alfven wave driven wind in red giants? 2) How can you justify the large expansion factor you used in Red Giants?

SuZUKI: 1) The collision frequency between ions and neutrals is shorter than the frequency of the Alfven waves I'm considering. So we can treat as 1-fluid for the ion frequency Alfven waves. But for high frequency waves, that effect is important. 2) In my opinion the large expansion is a consequence of the pressure balance between magnetic pressure and gas pressure. It is our working hypothesis. 